\title{
Zur Frage des Lichtwechsels des Sternes 190.1904 Cassiopeiae.
}

Diesen Stern hat A. Tass in A. N. 3991 und 4021 als veränderlich angezeigt. Safał́k benutzte denselben als Vergleichstern zu T Cassiopeiae. Aus seinen Beobachtungen habe ich die Größen für den verdächtigten Stern ermittelt und teile hier das Resultat meiner Untersuchung mit. Betracht :

Als Vergleichsterne kommen folgende Sterne in

$$
\begin{aligned}
& z=\mathrm{BD}+55^{\circ} 73\left(8^{\mathrm{m}} \mathrm{o}\right) 7^{\mathrm{m}} \cdot 7 \mathrm{o} \\
& d=\varnothing+5450(8.0) 8.25 \\
& \beta=\gg+5455(8.3) 8.30 \\
& \omega="+543^{6}(8.3) 7.60 \\
& \alpha=»+5554(8.3) \quad 7.85 \text { Refl. } \\
& "=» \text { 8.15 Refr. }
\end{aligned}
$$

Die nicht eingeklammerten Größen sind die aus den Beobachtungen Šafařkss abgeleiteten. (Refl. = Beob. am Reflektor, Refr. = Beob. am Refraktor).
Im ganzen liegen von dem Stern aus den Jahren 1883 bis 189095 Beobachtungen vor, welche nach den benutzten Instrumenten getrennt für I90.1904 Cassiopeiae $(v)$ folgende Größen ergeben :

$$
\begin{array}{ccc}
\text { Vgl.-Stern } & \text { Reflektor } & \text { Refraktor } \\
z & v=77^{\mathrm{m}} \cdot 95 \pm 0.95 & \\
d & 7.95 \pm 0.12 & 7^{\mathrm{m}} \cdot 95 \pm 0.9510 \\
\beta & 7.95 \pm 0.05 & \\
\alpha & 7.95 \pm 0.05 & 7.95 \pm 0.1 \mathrm{I} \\
\omega & 7.95 \pm 0.08 & 7.95 \pm 0.09
\end{array}
$$

Es ist demnach der Stern in der oben erwähnten Zeit von Safař́k stets in der Helligkeit $7^{\mathrm{m}} \cdot 95 \pm 0^{\mathrm{m}} \mathrm{ro}$ beobachtet worden, und es erscheint daher sehr wahrscheinlich, daß derselbe in dem Zeitraume der Beobachtungen konstantes Licht hatte.

Bamberg, Sternwarte, I 909 Juli 14.

L. Pracka.

\section{Eine ältere Beobachtung von $\gamma$ Pegasi.}

$\gamma$ Pegasi wurde als Vergleichstern zu " Ceti von Šafařk im Jahre r $88_{5}$ benutzt. Die Helligkeit wurde im Februar zu $4^{\mathrm{m}} \cdot \mathbf{2}$ im Potsdamer System geschätzt, also $\circ^{\mathrm{m}} \cdot 9$ schwächer als die Bestimmung von Müller und Kempf, und $\mathbf{I}^{\mathrm{m}} \cdot \mathbf{7} \mathrm{schwächer}$ als die von Pickering. Da die Beobachtung Šafar̀kks kaum $\pm 0^{\mathrm{m}} \mathbf{2}$ unsicher ist, ist der Stern der Veränderlichkeit verdächtig (Potsdam. Publ. Bd. I 7 pag. 287).

L. Pracka.

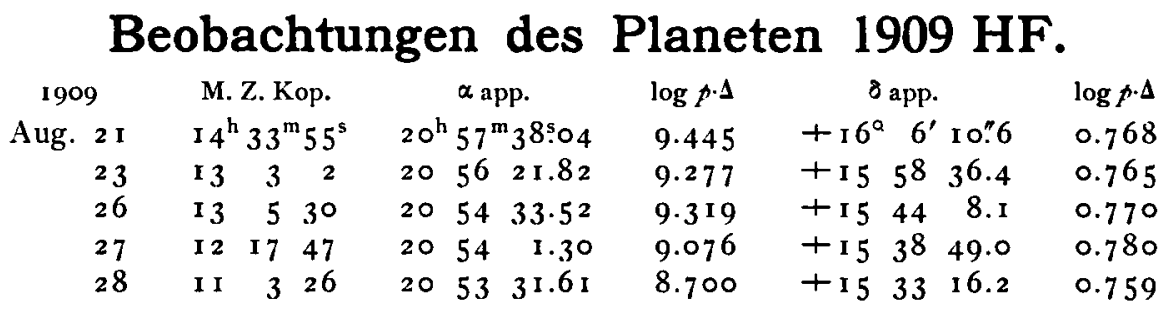

Kopenhagen, Universitäts-Sternwarte I 909 Sept. I.

C. F. Pechiile.

Mars. Une terre a été vu émergeant de la calotte polaire par I $20^{\circ}$. Elle ressemble à Argyre II qui se présente à $60^{\circ}$. Aonius Sinus.

Le canal Nectar est très large. Un autre très large également part de Solis Lacus pour rejoindre l'extrémité de

Observatoire d'Hem, rgog Sept. 2.

R. Fonckheere.

(365) Corduba. I 909 Aug. 23 I $2^{\mathrm{h}} 18^{\mathrm{m}} \cdot 8$ Kgst. $\alpha$ 1 $909.0=22^{\mathrm{h}} 33^{\mathrm{m}} 6 \delta_{1909.0}=+2^{\circ} 7^{\prime}$. Tägl. Bewegung: $-0^{\mathrm{m}} .8-9^{\prime}$. Gr. I I m.6. IV. Lorenz.

Neuer Planet 1909 HH. I 909 Aug. 25 I $3^{\mathrm{h}} 45^{\mathrm{m}} \mathrm{I}^{\mathrm{s}}$ Wien $\alpha$ app. $=23^{\mathrm{h}} 3^{\mathrm{m}} 53^{\mathrm{s}} \cdot 78(8.673) \delta$ app. $=-0^{\circ} 47^{\prime} 57^{\prime \prime} \cdot 3$ $\left(0.821\right.$ ). Tägl. Bewegung: $-34^{\mathrm{s}}-9 ! 3$. Gr. r2m. $2^{\mathrm{m}}$. . Palisa.

Personalnotiz. Mr. Ph. Fox, formerly at the Yerkes Observatory, assumed on Sept. I the directorship of the Dearborn Observatory, Evanston, Illinois, succeeding the late G. IW. Hough.

Inhalt zu Nr. 4353-54. S. W. Burnham. Double star measures. I33. - R. Fonckheere. Mars. I 59. - E. M. Antoniadi. Note sur la pâleur actuelle des taches de Mars. 16r. - A. Abetti. Osservazioni di Pianetini. 16r. - L. Pracka. Zur Frage des Lichtwechsels des Sternes 190.1904 Cassiopeiae. I63. - L. Pracka. Eine ältere Beobachtung von $\gamma$ Pegasi. I63. C. F. Pechïle. Beobachtungen des Planeten I909 HF. I63. - R. Fonckheere. Mars. I63. - Kleine Mitteilungen. 163. - Personalnotiz. I63. 